\title{
THE X-RAY QUIESCENCE OF SWIFT J195509.6+261406 (GRB 070610): AN OPTICAL BURSTING X-RAY BINARY?
}

\author{
N. Rea ${ }^{1}$, P. G. Jonker ${ }^{2,3,4}$, G. Nelemans ${ }^{4}$, J. A. Pons ${ }^{5}$, M. M. Kasliwal ${ }^{6}$, S. R. Kulkarni ${ }^{6}$, And R. WiJnands ${ }^{7}$ \\ ${ }^{1}$ Institut de Ciencies de l'Espai (CSIC-IEEC), Campus UAB, Facultat de Ciencies, Torre C5-parell, 2a planta, 08193, Bellaterra (Barcelona), Spain; rea@ ieec.uab.es \\ ${ }^{2}$ SRON-Netherlands Institute for Space Research, Sorbonnelaan 2, 3584 CA Utrecht, The Netherlands \\ ${ }^{3}$ Harvard-Smithsonian Center for Astrophysics, 60 Garden Street, Cambridge, MA 02138, USA \\ ${ }^{4}$ Department of Astrophysics, Radboud University Nijmegen, IMAPP, P.O. Box 9010, 6500 GL, Nijmegen, The Netherlands \\ ${ }^{5}$ Departament de Fisica Aplicada, Universitat d'Alacant, Ap. Correus 99, 03080 Alacant, Spain \\ ${ }^{6}$ Department of Astronomy, California Institute of Technology, Mail Stop 105-24, Pasadena, CA 91125, USA \\ ${ }^{7}$ University of Amsterdam, Astronomical Institute “Anton Pannekoek," Postbus 94249, 1090 GE, Amsterdam, The Netherlands \\ Received 2010 November 11; accepted 2011 January 18; published 2011 February 15
}

\begin{abstract}
We report on an $\sim 63 \mathrm{ks}$ Chandra observation of the X-ray transient Swift J195509.6+261406 discovered as the afterglow of what was first believed to be a long-duration gamma-ray burst (GRB 070610). The outburst of this source was characterized by unique optical flares on timescales of second or less, morphologically similar to the short X-ray bursts usually observed from magnetars. Our Chandra observation was performed $\sim 2$ years after the discovery of the optical and X-ray flaring activity of this source, catching it in its quiescent state. We derive stringent upper limits on the quiescent emission of Swift J195509.6+261406, which argues against the possibility of this object being a typical magnetar. Our limits show that the most viable interpretation on the nature of this peculiar bursting source is a binary system hosting a black hole or a neutron star with a low-mass companion star $\left(<0.12 M_{\odot}\right)$ and with an orbital period smaller than a few hours.
\end{abstract}

Key words: stars: magnetic field - X-rays: individual (SWIFT J195509.6+261406) - X-rays: stars

Online-only material: color figures

\section{INTRODUCTION}

On 2007 June 10 the Swift Burst Alert Telescope (BAT) triggered on GRB 070610, a typical long-duration GRB (gammaray burst; see Gehrels et al. 2007 for a recent review), with an $\sim 4.6$ s high-energy prompt emission (Pagani et al. 2007; Tueller et al. 2007). Follow-up soft X-ray observations with the Swift X-Ray Telescope (XRT) started soon after the event, discovering only one variable X-ray source within the BAT error circle, namely, Swift J195509.6+261406 (hereafter Swift J1955; Kasliwal et al. 2008). This transient X-ray source was very different from what expected for the X-ray afterglow of a long GRB: it was decreasing in flux rather slowly, and it showed a strong X-ray flaring activity.

The source became undetectable by Swift-XRT on 2007 June 29, ranging from a $0.5-10 \mathrm{keV}$ flux of $\sim 10^{-9}$ to $<10^{-12} \mathrm{erg} \mathrm{s}^{-1} \mathrm{~cm}^{-2}$ in 19 days. While in outburst, Swift J1955 had an X-ray spectrum that could be described by a rather hard power law corrected for the photoelectric absorption $\left(N_{\mathrm{H}}=7 \times 10^{21} \mathrm{~cm}^{-2}\right.$ and $\left.\Gamma=1.7\right)$. Due to spatial and temporal coincidence (it was the only transient source in the BAT error circle), GRB 070610 and the X-ray transient Swift J1955 have been associated with high probability (Kasliwal et al. 2008).

The most interesting and peculiar features of this transient source came from optical and infrared observations. Many telescopes, triggered by the GRB-like event, promptly observed the position of Swift J1955 during the outburst. A highly variable optical and infrared counterpart was observed, showing large flares for about 11 days after the GRB-like event, when it went back to quiescence. These large flares were characterized by a very short timescale: during the largest flare, the source increased its optical flux by more than a factor of 200 in less than $4 \mathrm{~s}$. Furthermore, a broad quasi-periodic oscillation was observed in the optical band at $\sim 0.16 \mathrm{~Hz}$ (Stefanescu et al. 2008).
The source distance was constrained by several different methods to be within 3.7-10 kpc (mainly red clump study, and detailed measurements of the absorption column in the millimeter waveband; Castro-Tirado et al. 2008). Furthermore, the stringent optical and IR limits derived in the quiescent level $\left(H>23 ; R>26.0\right.$ and $i^{\prime}>24.5$; Kasliwal et al. 2008; Castro-Tirado et al. 2008) constrain the type of any companion star to either a main-sequence star with spectral type later than M5V (which means a mass $<0.12 M_{\odot}$ ) or to a semi-degenerate hydrogen poor star (Castro-Tirado et al. 2008).

The large variations of its optical and infrared counterpart during the decay to quiescence, its distance and Galactic nature, set this transient apart from the typical optical afterglows of long-duration GRBs (see Liang et al. 2007 for a recent review).

The resemblance of the optical bursts of Swift J1955 with the short X-ray bursts from magnetars (see Mereghetti 2008 for a recent review) led to the idea of a new kind of X-ray and optical transient event in a Galactic magnetar (Castro-Tirado et al. 2008; Stefanescu et al. 2008). On the other hand, its X-ray flaring activity was also proposed to resemble the emission of the fast X-ray nova V4641 Sgr (Markwardt et al. 2008; Kasliwal et al. 2008), an unusual $9 M_{\odot}$ black hole in orbit with a 5-8 $M_{\odot}$ B9 III companion star (in't Zand et al. 2000; Orosz et al. 2001)

In this Letter, we present the results of an $\sim 63 \mathrm{ks}$ Chandra observation of Swift J1955 (see Section 2) aimed at unveiling its $\mathrm{X}$-ray properties during quiescence (see Section 3) and compare them with the current quiescent levels of the magnetar and X-ray binary populations (see Section 4).

\section{OBSERVATION AND DATA ANALYSIS}

The Chandra X-Ray Observatory observed Swift J1955 for $\sim 63$ ks with the Advanced CCD Imaging Spectrometer (ACIS) instrument (ObsID 10042) from 2009 August 3 16:09:57 to August 4 09:55:59 (Terrestrial Time) in VERY FAINT (VF) 
timed exposure imaging mode. The source was positioned on the back-illuminated ACIS-S3 CCD at the nominal target position (R.A.: 1955 09.653, decl.: +26 $1405.84 \pm 0$ '.27; J2000), and we used a sub-array of $1 / 8$ leading to a time resolution of 1.14 s. Standard processing of the data was performed by the Chandra X-Ray Center to Level 1 and Level 2 (processing software DS 8.0). The data were reprocessed using the CIAO software (version 4.1.2). We used the latest ACIS gain map, and applied the time-dependent gain and charge transfer inefficiency corrections. The data were then filtered for bad event grades and only good time intervals were used. No high background events were detected, resulting in a final on-time exposure of $62.732 \mathrm{ks}$.

\section{RESULTS}

We did not detect any X-ray source at the position of the optical counterpart to Swift J1955 (Kasliwal et al. 2008; Castro-Tirado et al. 2008). In particular, we detected no 0.3-10 keV photons within a $1^{\prime \prime}$ circle centered on the optical position. We took a $95 \%$ upper limit of three photons (Gehrels 1986) and inferred a $4.78 \times 10^{-5}$ counts $^{-1}$ upper limit on the $\mathrm{X}$-ray quiescent count rate of Swift J1955.

Using PIMMS $^{8}$ we estimated the $95 \%$ upper limit on the source flux assuming: (1) an absorbed power-law spectrum similar to the outburst spectral energy distribution $\left(N_{\mathrm{H}}=7 \times\right.$ $10^{21} \mathrm{~cm}^{-2}$ and $\Gamma=1.7$; Kasliwal et al. 2008) and (2) a quiescent thermal spectrum with the same $N_{\mathrm{H}}=7 \times 10^{21} \mathrm{~cm}^{-2}$ and $k T=$ $0.3 \mathrm{keV}$, typical of a magnetar in quiescence (see, i.e., Muno et al. 2008; Bernardini et al. 2009). We derived a 95\% upper limit on the observed $0.3-10 \mathrm{keV}$ absorbed (unabsorbed) flux of $6.2(9.6) \times 10^{-16} \mathrm{erg} \mathrm{s}^{-1} \mathrm{~cm}^{-2}$ and $2.1(6.6) \times 10^{-16} \mathrm{erg} \mathrm{s}^{-1}$ $\mathrm{cm}^{-2}$ under the power-law and blackbody spectral assumptions, respectively. Note that these spectral decompositions, and the derived flux limits, also comprise the typical values for quiescent $\mathrm{X}$-ray binaries.

\section{DISCUSSION}

In this Letter, we present a deep X-ray observation of Swift J1955 during the quiescent state. Before entering in a detailed discussion of our X-ray limit on the quiescent emission of this optical bursting transient, we first need to discuss the current estimates on the source distance.

Castro-Tirado et al. (2008) studied in detail the distance issue, first taking ${ }^{12} \mathrm{CO}$ and $\mathrm{H}$ i spectra, and then deriving the extinction versus distance distribution in the Swift J1955 line of sight based on the red clump method (see also Lopez-Corredoira et al. 2002; Durant \& van Kerkwijk 2006). From the millimeter and centimeter spectra, they derived a Galactic column density in the direction of this source of $N_{\mathrm{H}}=N_{\mathrm{HI}}+2 N_{\mathrm{H} \text { II }}=$ $(14.1 \pm 2.0) \times 10^{21} \mathrm{~cm}^{-2}$, half of which is accounted for by a molecular cloud at $3.7 \mathrm{kpc}$ (considered as a lower limit on the distance of Swift J1955). Comparing the Galactic $N_{\mathrm{H}}$ with the one derived from fitting the X-ray spectrum during outburst $\left(N_{\mathrm{H}}=7.2_{-2}^{+3} \times 10^{21} \mathrm{~cm}^{-2}\right.$; Kasliwal et al. 2008), ${ }^{9}$ Swift J1955 is expected to be located at $\sim 4-5 \mathrm{kpc}$. Similar results have been derived from the red clump method, from which a distance of $\sim 4 \mathrm{kpc}$ could be inferred (Castro-Tirado et al. 2008). Unfortunately, the upper limit on the source distance is not very well constrained, although all methods used would

\footnotetext{
8 http://heasarc.gsfc.nasa.gov/Tools/w3pimms.html

9 Note that this is derived from an absorbed power-law fit (Kasliwal et al. 2008), hence it might be an overestimate on the $N_{\mathrm{H}}$ of the source.
}

place the source $\sim 4-5 \mathrm{kpc}$. The main problem to assess a distance error bar is that the Galactic plane in the direction of Swift J1955 extends only until $\sim 5 \mathrm{kpc}$, behind which there is the bulge, with its intrinsically lower column density. This makes it extremely hard to define an upper limit to the distance, since in the bulge a column density versus distance relation is not well defined. Hereafter, we will discuss our results assuming a distance range of 3.7-10 kpc, considering the farthest limit in the Milky Way as a distance upper limit.

The $95 \%$ upper limit on the quiescent X-ray luminosity of Swift J1955 is $2.8 \times 10^{30} \mathrm{~d}_{5 \mathrm{kpc}}^{2}$ erg s$^{-1}$ or $1.9 \times 10^{30} \mathrm{~d}_{5 \mathrm{kpc}}^{2} \mathrm{erg} \mathrm{s}^{-1}$ for the power-law or blackbody models, respectively (see also Section 3). Considering the whole 3.7-10 kpc range (see above), the derived quiescent $\mathrm{X}$-ray luminosity range is between 1.5 and $11.4 \times 10^{30} \mathrm{erg} \mathrm{s}^{-1}$ or 1.0 and $7.9 \times 10^{30} \mathrm{erg} \mathrm{s}^{-1}$, again assuming a power law or a blackbody, respectively.

\subsection{On the Magnetar Interpretation}

The discovery of fast optical bursts in Swift J1955 led a few authors to claim the magnetar nature of this source (Castro-Tirado et al. 2008; Stefanescu et al. 2008), based on the similarity of the optical light curve with the typical short $\mathrm{X}$-ray bursts from magnetars. However, neither a complete understanding of the physical processes involved in these optical bursts nor other magnetar-like features (slow spin period, presence of magnetar-like X-ray bursts, etc.) help in characterizing this source as a high magnetic field neutron star. Furthermore, the GRB-like event emitted by this source is at variance with any other flaring activity detected thus far from magnetars (see Mereghetti 2008 for a review).

For an isolated neutron star, a luminosity limit of $\sim 10^{30}$ $10^{31} \mathrm{erg} \mathrm{s}^{-1}$ would necessarily imply a source older than $5 \times$ $10^{5}$ years, for any cooling model or equation of state (Lattimer \& Prakash 2001; Yakovlev \& Pethick 2004). This age limit would make Swift J1955 two order of magnitudes older than the bulk of magnetars (Mereghetti 2008), in line only with the old low-B field SGR 0418+5729 (Rea et al. 2010). Furthermore, the former value can be considered a lower limit on the age, since for the cooling models for high magnetic field neutron stars (Pons et al. 2009; Aguilera et al. 2008, 2009), and the heat released by the recent outburst, these will go in the direction of predicting a larger age. In particular, for a typical cooling neutron star, the presence of a high magnetic field causes a much brighter source at the same age.

Assuming a neutron star with $1.4 M_{\odot}$, we have used the cooling code of Pons et al. (2009) to simulate the cooling decay of a magnetar with three different initial magnetic field values (see Figure 1, left panel). To reach our upper limit on the luminosity of $L_{\mathrm{x}} \sim 10^{31} \mathrm{erg} \mathrm{s}^{-1}$ for a magnetar-like magnetic field of $B>10^{14} \mathrm{G}$ at birth, the source should be now older than $120 \mathrm{Myr}$ (see the dashed line in Figure 1, left panel), and having now a magnetic field of a few $\times 10^{13}$, hence below the magnetar regime. On the other hand, the magnetic field and the strong internal helicity, supposed to produce short bursts and outburst activity in magnetars, should have been largely dissipated at these old times and low field (see Figure 1, right panel). Taking at face value the luminosity currently measured for typical quiescent magnetars, the luminosity we derive is fainter than the faintest magnetar in quiescence (SGR 0418+5729: $\sim 6 \times$ $10^{31} \mathrm{erg} \mathrm{s}^{-1}$; Rea et al. 2010). The possibility of Swift J1955 being a case similar to the low-B field SGR $0418+5729$ is intriguing; however, the large GRB-like flare detected from the former would be hardly explainable within the scenario of an 

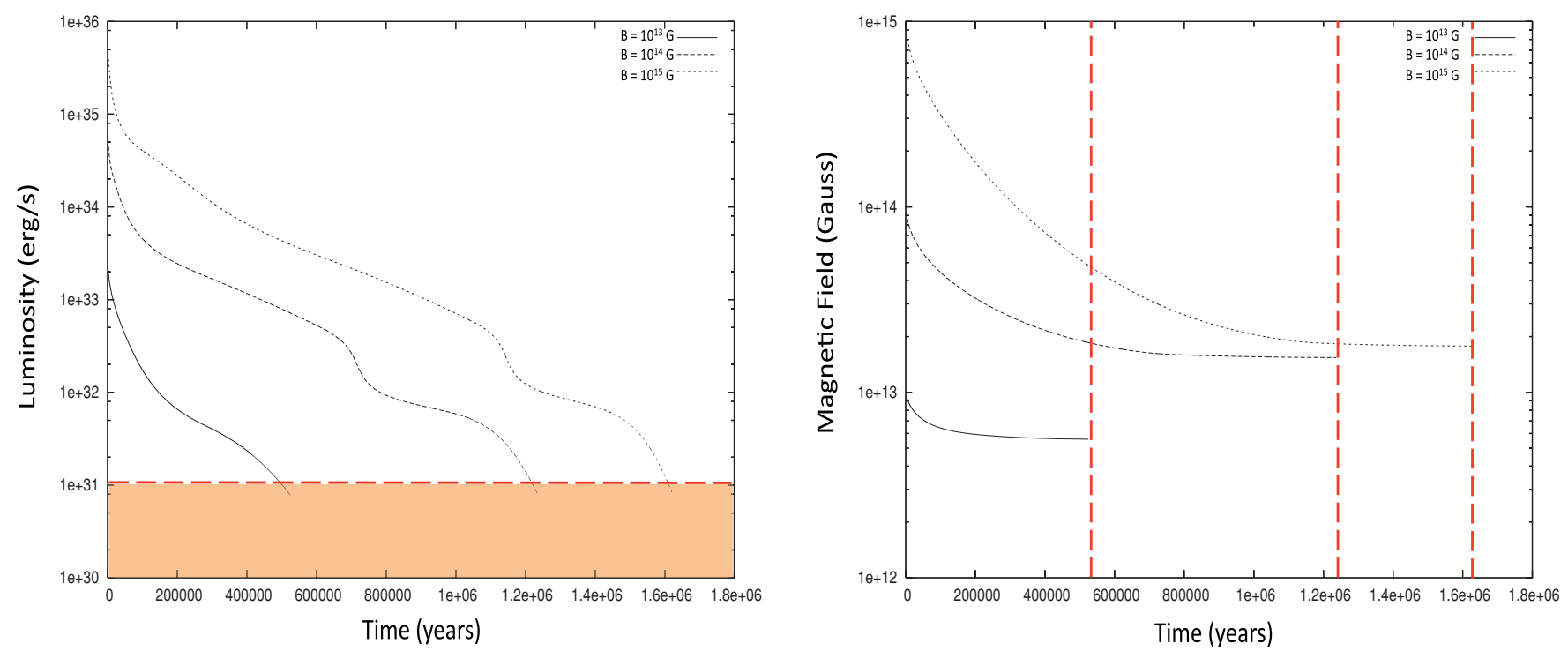

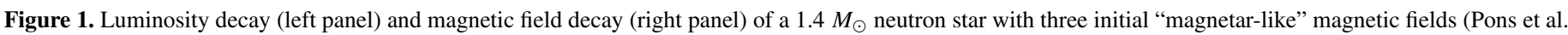

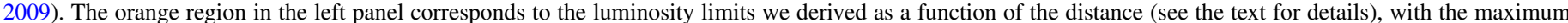

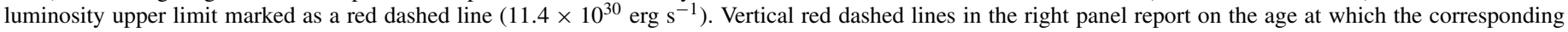
cooling magnetar would reach this maximum luminosity upper limit.

(A color version of this figure is available in the online journal.)

old magnetar releasing its last bit of internal magnetic energy through weak sporadic bursts, as for SGR 0418+5729.

Furthermore, unless the source is in the Galactic halo at a distance of $>10 \mathrm{kpc}$, it is also dimmer than the luminosity of any X-Ray Dim Isolated Neutron Star known to date $\left(\sim 10^{31}\right.$ $\mathrm{erg} \mathrm{s}^{-1}$; Turolla 2009), making the possible association of this object with any of those classes rather unlikely.

\subsection{An X-ray Binary System}

A more plausible scenario is the X-ray binary nature of Swift J1955. In the binary case, optical and infrared observations during quiescence could put a limit on the companion mass of $<0.12 M_{\odot}$ (with a spectral type later than M5V), or being a semi-degenerate hydrogen poor star (Castro-Tirado et al. 2008). The occurrence of the X-ray outburst implies that the low-mass star orbiting the compact object should be (close to) filling its Roche lobe. Assuming it fills its Roche lobe, and it is a mainsequence star, this gives a unique relation between the orbital period of the system and the mass of the companion star (see Equation (4.11) in Frank et al. 2002).

Given the limits on the companion star, the system orbital period is constrained to be shorter than $1.2 \mathrm{hr}$ if the star is on the main sequence. Obviously, shorter periods are also allowed in the ultracompact binary case with an H-poor white dwarf. Another viable possibility is a hot brown dwarf companion star, in which case the orbital period of the system is constrained to be shorter than a few hours (Bildsten \& Chakrabarty 2001).

$\mathrm{X}$-ray observations of low-mass X-ray binaries during quiescence have empirically shown that neutron star and black hole binaries, with the same orbital periods, show different quiescent luminosities (see, e.g., Lasota 2008 and Figure 2). This observational evidence might have a few interpretations. One possibility is that neutron stars' hot surface makes them always brighter during quiescence than a black hole, where supposedly an event horizon is instead in place (Narayan et al. 1997). Another possibility might instead be the different accretion energy release mechanism, with black holes releasing more energy through their radio jets rather than in the X-ray band (Fender et al. 2003). In Figure 2, we plot the quiescent X-ray luminosity of all binary neutron stars and black holes for which this has been measured, and an orbital period or a limit on it could be derived (see also Garcia et al. 2001; Kong et al. 2002; Jonker et al. 2006; Lasota 2008). The orange region in Figure 2 is the quiescent luminosity space limit we derived for Swift J1955 considering the 3.7-10 kpc distance range and two different spectral models (see above). We also plot the luminosity limits considering the most plausible distance of $5 \mathrm{kpc}$ and for the larger distance of $10 \mathrm{kpc}$ (dashed and dot-dashed red and gray horizontal lines, respectively).

In rest of the discussion, we attempt to distinguish between the neutron star and black hole hypotheses.

\subsubsection{A Neutron Star System}

The quiescent luminosity of a neutron star after $\sim 2$ years from an outburst is strongly dependent on the outburst history. The longer the outburst activity the longer the cooling will take to reach the pre-outburst luminosity level (Brown et al. 1998). In our case, there are no previous outbursts or bursts recorded from Swift J1955 in the past years, hence the heating dumped on the putative neutron star surface is very little. This small heating can explain the fast decrease in luminosity (by $\sim 7$ orders of magnitudes) from the GRB 070610 event till 2009 August when our deep X-ray upper limits are derived.

It is evident from Figure 2 that at $\sim 5 \mathrm{kpc}$, Swift J1955 would be an ultracompact binary system, being too faint in quiescence for a neutron star accreting from a main-sequence star or a brown dwarf. In this scenario, this system might be similar to the ultracompact binary H1905+00 (namely, the upper limit reported on the bottom left of Figure 2; Jonker et al. 2006, 2007). On the other hand, if the putative neutron star is instead at about $10 \mathrm{kpc}$, then it might still be in orbit with a main-sequence star or a brown dwarf, and the binary should have an orbital period shorter than a few hours (Frank et al. 2002; Bildsten \& Chakrabarty 2001). 


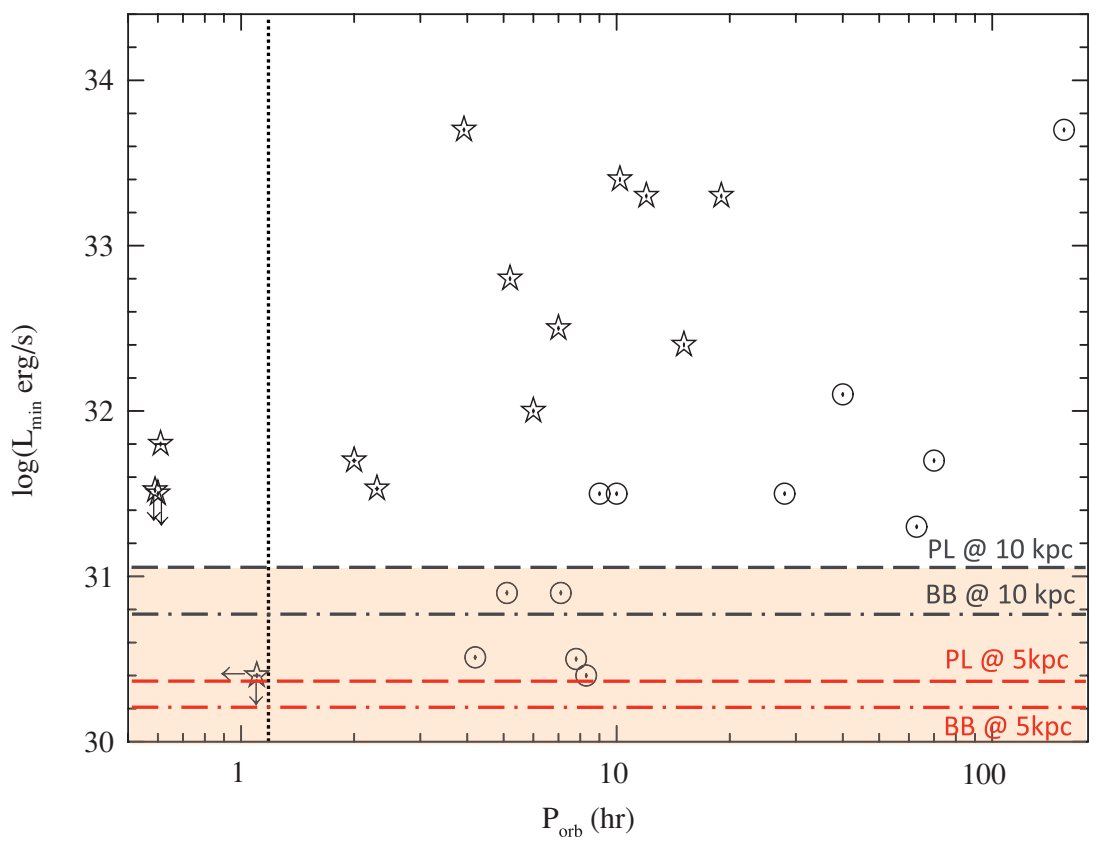

Figure 2. Quiescent $0.5-10 \mathrm{keV}$ luminosity vs. $P_{\text {orb }}$ for neutron star (stars) and black hole (circles) X-ray binaries (adapted from Lasota 2008; Garcia et al. 2001; Jonker et al. 2007; Degenaar et al. 2009; plus the addition of archival X-ray observations). Vertical arrows represent upper limits on the X-ray quiescent emission. Horizontal dashed and dot-dashed lines represent our 95\% upper limits on the X-ray quiescent luminosity of Swift J1955 for a power-law and a blackbody spectral model, respectively, and for a 5 and a $10 \mathrm{kpc}$ distance (see the text for details). The orange shadowed region is the allowed luminosity space considering the whole 3.7-10 kpc distance range, again for the two assumed spectral models. The vertical line at $1.2 \mathrm{hr}$ is the upper limit on the orbital period of Swift J1955 for a main-sequence companion star (see the text more details).

(A color version of this figure is available in the online journal.)

That said, it is important to note that neutron star accreting systems have not been seen showing large optical flares beside the optical counterpart to Type I or Type II bursts that are orders of magnitude fainter and with longer timescales than those observed in Swift J1955 (Kasliwal et al. 2008; Stefanescu et al. 2008; Castro-Tirado et al. 2008).

\subsubsection{A Black Hole System}

Large X-ray and optical flares, on several timescales, are an ubiquitous characteristic of black hole binaries. In particular, transient low-mass X-ray binaries hosting a black hole candidate undergo very dramatic $\mathrm{X}$-ray and optical outbursts and have long periods (even decades) of quiescence. However, sub-second timescale optical bursts such as in Swift J1955 (Stefanescu et al. 2008) were never observed before in any black hole binary (nor any other astronomical source either). Optical flares on several timescales down to minutes were reported, i.e., for A0620-00 (Hynes et al. 2003a), XTE J1118+480 (Hynes et al. 2003b), and GRS 1124-684 and Cen X-4 (Shahbaz et al. 2010). A somewhat similar case might be GX 339-4, the typical black hole candidate, which showed fast optical variability shorter than a second during a recent outburst (Gandhi et al. 2010). Similarities can also be found with the X-ray nova V4641 Sgr (as suggested by Kasliwal et al. 2008), although in this case the relatively massive companion star can introduce a somehow different physical process than in Swift J1955.

Apart from the peculiar optical behavior, all the other observational characteristics observed from Swift J1955 are in line with what already observed from black hole binaries: energetic X-ray flares, fast decay into quiescence, optical QPOs at $0.16 \mathrm{~Hz}$, and extremely faint X-ray quiescence luminosities (see Figure 2).

The possibility of Swift J1955 being a black hole in an ultracompact binary system is very interesting, as it would be the first ever discovered. Although very speculative, it might be possible that the unique optical behavior of this source is indeed reflecting the first of such systems, the emission of which is still largely unknown.

\section{CONCLUSIONS}

We derived deep upper limits with Chandra on the X-ray quiescent emission of the optical bursting transient Swift J1955. We showed that a magnetar scenario is very unlikely: the source is too faint in quiescence for any realistic scenario of magnetar cooling. We suggest that Swift J1955 is most likely an X-ray binary, hosting a black hole or a neutron star with an orbital period faster than a few hours, possibly in an ultracompact system. High-time resolution optical observations of X-ray binaries during outburst might reveal energetic optical flares, a peculiarity that Swift J1955 does not share yet with any other source.

N.R. is supported by a Ramón y Cajal fellowship through Consejo Superior de Investigaciones Cientfícas, by grants AYA2009-07391 and SGR2009-811, and thanks to P. Casella for useful discussions on the optical variability in black hole binaries. P.G.J. and G.N. acknowledge support from a VIDI grant from the Netherlands Organization for Scientific Research.

\section{REFERENCES}

Aguilera, D. N., Pons, J. A., \& Miralles, J. A. 2008, A\&A, 486, 255

Aguilera, D. N., et al. 2009, Phys. Rev. Lett., 102, 1101

Bernardini, F., et al. 2009, A\&A, 498, 195

Bildsten, L., \& Chakrabarty, D. 2001, ApJ, 557, 292

Brown, E. F., Bildsten, L., \& Rutledge, R. E. 1998, ApJ, 504, L95

Castro-Tirado, A. J., et al. 2008, Nature, 455, 506

Degenaar, N., et al. 2009, MNRAS, 396, L26 
Durant, M., \& van Kerkwijk, M. K. 2006, ApJ, 650, 1070

Fender, R., Gallo, E., \& Jonker, P. G. 2003, MNRAS, 343, L99

Frank, J., King, A., \& Raine, D. J. 2002, Accretion Power in Astrophysics (Cambridge Univ. Press)

Gandhi, P., et al. 2010, MNRAS, 407, 2166

Garcia, M. R., et al. 2001, ApJ, 553, L47

Gehrels, N. 1986, ApJ, 303, 336

Gehrels, N., et al. 2007, New J. Phys., 9, 37

Hynes, R. I., et al. 2003a, MNRAS, 340, 447

Hynes, R. I., et al. 2003b, MNRAS, 345, 292

in't Zand, J. J. M., et al. 2000, A\&A, 357, 520

Jonker, P. G., et al. 2006, MNRAS, 368, 1803

Jonker, P. G., et al. 2007, ApJ, 665, L147

Kasliwal, M. M., et al. 2008, ApJ, 678, 1127

Kong, A., et al. 2002, ApJ, 570, 277

Lattimer, J. M., \& Prakash, M. 2001, ApJ, 550, 426

Lasota, J-P. 2008, New Astron. Rev., 51, 752
Liang, E., et al. 2007, ApJ, 670, 565

Lopez-Corredoira, M., Cabrera-Lavers, A., Garzon, F., \& Hammersley, P. L. 2002, A\&A, 394, 883

Markwardt, C. B., et al. 2008, ATel, 1102

Mereghetti, S. 2008, A\&AR, 15, 225

Muno, M. P., et al. 2008, MNRAS, 378, L44

Narayan, R., Garcia, M. R., \& McClintock, J. 1997, ApJ, 478, L79

Orosz, J. A., et al. 2001, ApJ, 555, 489

Pagani, C., et al. 2007, GCN Circ., 6489, 1

Pons, J. A., Miralles, J., \& Geppert, U. 2009, A\&A, 496, 207

Rea, N., et al. 2010, Science, 330, 944

Shahbaz, T., et al. 2010, MNRAS, 403, 2167

Stefanescu, A., et al. 2008, Nature, 455, 503

Tueller, M., et al. 2007, GCN Circ., 6491, 1

Turolla, R. 2009, in Neutron Stars and Pulsars, ed. W. Becker (Astrophysics and Space Science Library, Volume 357; Berlin: Springer), 141

Yakovlev, D. G., \& Pethick, C. J. 2004, AR\&A, 42, 169 